\title{
Estimación y análisis de umbrales críticos de lluvia para la ocurrencia de avenidas torrenciales en el Valle de Aburrá (Antioquia)
}

\author{
$\checkmark$ Luis Ángel Guerrero Hoyos ${ }^{1}$ \\ EDIER ARISTIZÁBAL GIRALDO ${ }^{1}$
}

\section{Resumen}

En Colombia entre los años 1914 y 2015, se han presentado 1.139 avenidas torrenciales con un saldo trágico de 2.195 víctimas mortales. Eventos como los ocurridos en Salgar (2015) y Mocoa (2017) señalan la necesidad de implementar sistemas de alerta temprana en aquellas áreas susceptibles que han sido densamente pobladas. En el presente trabajo se estudia el caso del valle de Aburrá; se proponen umbrales de lluvia críticos como detonantes de avenidas torrenciales para un sistema de alerta temprana. Para la definición de umbrales se utilizó el método del RTI (Índice de Lluvia Detonante), desarrollado en Taiwán. El método utiliza datos de lluvia horarios de 15 estaciones localizadas dentro del valle y el inventario de avenidas registradas en el DesInventar. A partir de estas series de datos se encontraron 1.784 eventos de lluvia detonantes potenciales entre 1994-2016, con un promedio de intensidad máxima de 31,2 mm/h. Los resultados arrojan valores de RTI críticos de 2.268, 2.734, 3.128, $3.337 \mathrm{~mm}^{2} / \mathrm{h}$ para 1, 3, 7 y 15 días de lluvia antecedente respectivamente; con la intensidad máxima promedio se obtuvo un umbral crítico de lluvia antecedente acumulada de 76, 91, 104, $111 \mathrm{~mm}$ para 1, 3, 7 y 15 días de lluvia antecedente, respectivamente.

Palabras clave: umbrales, avenidas torrenciales, alerta temprana, lluvia, Valle de Aburrá

1 Universidad Nacional de Colombia. Medellín, Colombia.

Autor de correspondencia: Guerrero Hoyos, L.Á. (Luis Ángel): Cra 85 \#65a-50 Apto 9810 (Unidad Camino del Bosque). Medellín, Colombia Teléfono: (+57)3005446497. Correo electrónico: laguerreroh@unal.edu.co
Historia del artículo:

Artículo recibido: 20-XII-2018 / Aprobado: 30-V-2019

Disponible online: 3 de julio de 2019

Discusión abierta hasta marzo de 2021 


\title{
Critical Rainfall Threshold Analysis And Estimation For Flash Flood Occurrence In Aburrá Valley (Antioquia)
}

\begin{abstract}
In Colombia between 1914 and 2015, there have occurred 1.139 flash floods leaving a tragic balance with 2.195 mortal victims. Recent cases: Salgar (2015) and Mocoa (2017) show the urgent necessity of establishing an early warningalarm system for those torrential phenomena susceptible areas which have been densely populated. This job studies the Aburrá Valley case, where have recurrently presented torrential events, and puts forward critical threshold like a flash floods detonating to implementing these on an early warning-alarm system. Critical threshold were defined by the RTI (Rainfall Triggering Index) methodology, developed in Taiwan. This method uses hourly rainfall data of 15 rain gauges located inside of Aburrá Valley and flash floods inventory registered by DesInventar. From this data, it was established 1.784 potential detonating rainfall events between 1994 and 2016, with and maximum intensity average of 31,2 mm/h. Results found critical RTI values of 2.268, 2.734, 3.128 and $3.337 \mathrm{~mm}^{2} / \mathrm{h}$ for $1,3,7$ and 15 days of antecedent rainfall respectively. Used the maximum intensity average is obtained a critical threshold of cumulated antecedent rainfall of 76 , 91, 104 and $111 \mathrm{~mm}$ for $1,3,7$ and 15 days of antecedent rainfall respectively.
\end{abstract}

Keywords: threshold, flash floods, early waning, rainfall, Aburrá Valley.

\section{Estimação e análises dos limiares críticos de chuva para a ocorrência de inundações torrenciais no Vale do Aburrá (Antioquia)}

\section{Resumo}

Na Colômbia entre os anos de 1914 e 2015, foram apresentadas 1.139 inundações torrenciais com um saldo trágico de 2.195 mortes. Os recentes casos de Salgar (2015) e Mocoa (2017) mostram a necessidade urgente de estabelecer sistemas de aviso antecipado nas áreas suscetíveis que foram densamente povoadas. Este artigo estuda o caso do Vale do Aburrá onde tem sido recorrentes este tipo de eventos. 0 estudo propõe limiares de chuva críticos como detonante das inundações torrenciais para sua implementação como parte de um sistema de aviso antecipado. Para a definição dos limiares foi utilizado o método do RTI (indicador de chuva detonante), que foi criado em Taiwan. 0 método é implementado com dados de precipitações de 15 estações e o inventário de inundações registradas no DesInventar. Nas séries de dados foram identificados 1.784 eventos potenciais de chuva detonante no Vale do Aburrá no período entre 1994 e 2016 , com uma intensidade máxima média de 31,2 mm / h. Os resultados mostram valores críticos de RTI 2268, 2734, 3128, $3337 \mathrm{~mm} 2$ / h durante 1, 3, 7 e 15 dias de chuva antecedente, respectivamente, para os quais com a intensidade máxima média se tem um limiar critico de chuva antecedente acumulada de 76, 91, 104, 111 mm durante 1, 3, 7 e 15 dias de chuva antecedente acumulada, respectivamente.

Palavras-chave: limiares, avenidas torrenciais, aviso antecipado, chuva, Vale do Aburrá. 


\section{Introducción}

Las avenidas torrenciales son una mezcla de agua y sedimentos en diferentes proporciones que se desplazan a lo largo de cauces a grandes velocidades en cuencas de montaña de áreas reducidas (Díez Herrero, Laín Huerta and Llorente Isidro, 2008; Borga et al., 2010, 2014; Destro et al., 2018). Debido a las causas diversas que originan las avenidas torrenciales, en Colombia han sido clasificadas desde flujos gravitacionales de ladera tipo movimientos en masa (INGEOMINAS - CVC, 2001; PMAGMA, 2007) hasta flujos hidrológicos tipo crecientes súbitas (FONDO DE PREVENCIÓN Y ATENCIÓN DE EMERGENCIAS (FOPAE), 2011; Ministerio de Medio Ambiente y Desarrollo, 2014). Las avenidas torrenciales se presentan generalmente como el resultado de un fenómeno en cascada, donde la lluvia juega un papel fundamental, pero no siempre detonante. Entre los mecanismos detonantes se encuentran eventos de lluvia intensos o incluso lluvias acumuladas antecedentes, enjambre de movimientos en masa detonados por lluvias o sismos, rotura súbita de presas naturales generadas por deslizamientos o depósitos glaciares, presas de origen antrópico, o fusión repentina de glaciares por actividad volcánica (Takahashi, 1991; RM Iverson, 1997; Sterling and Slaymaker, 2007; Díez Herrero, Laín Huerta and Llorente Isidro, 2008). Según (Díez Herrero, Laín Huerta and Llorente Isidro, 2008), las avenidas torrenciales se diferencian de las crecidas ya que el aumento del caudal se presenta de forma súbita y desde aguas arriba del observador, y de las avenidas súbitas por que se presentan en cauces torrenciales de sistemas montañosos.

De acuerdo con la reología del flujo como consecuencia de la proporción de agua y sedimentos (O'Brien and Julien, 1985; Pierson and Costa, 1987), y el tamaño de los sedimentos (COUSSOT and MEUNIER, 1996; RM Iverson, 1997), las avenidas torrenciales pueden clasificarse en flujos de una fase denominados generalmente flujos de escombros, y flujos de dos fases denominados como inundaciones de escombros (Aulitzky, 1980; Hungr, 2001; Sterling and Slaymaker, 2007; Hungr, Leroueil and Picarelli, 2014). Los flujos torrenciales tipo flujo de escombros corresponden a flujos viscosos no newtonianos que se desplazan como pulsos: tienen concentraciones de sedimentos que superan el $50 \%$ con capacidad de transportar grandes bloques de roca por suspensión; se presentan generalmente en drenajes de primer y segundo orden en cuencas que alcanzan sólo unos pocos kilómetros cuadrados con caudales picos extremos. Entre tanto los flujos torrenciales tipo inundación de escombros corresponden a flujos viscosos que no presentan pulsos; presentan reducido contenido de sedimentos finos y considerable capacidad de transportar grandes bloques por arrastre; se presentan en cuencas más grandes con caudales picos comparables a las inundaciones y los depósitos se extienden sobre distancias mayores y áreas de menor pendiente (Aulitzky, 1980; Hungr et al., 2001; Sterling and Slaymaker, 2007; Hungr, Leroueil and Picarelli, 2014). Sin embargo, aunque en teoría es posible diferenciar los tipos de flujos torrenciales, en realidad existe un continuo entre deslizamientos, flujos de escombros canalizados, inundaciones de escombros y crecientes súbitas, ya que los límites entre ellos no son tajantes, y en la naturaleza se presentan fenómenos que constan simultáneamente de varios de estos tipos de flujos torrenciales en términos espaciales y temporales (O’Brien and Julien, 1985; Costa, 1987; Jakob et al., 2006; Borga et al., 2014).

Aunque en los Andes colombianos se han registrado flujos torrenciales por diferentes causas, el gran porcentaje de eventos están asociados a lluvias de alta intensidad y corta duración en cuencas pequeñas de montaña y fuerte relieve relativo, donde el proceso de trasferencia de escorrentía es predominantemente superficial, generando una rápida respuesta hidrológica de la cuenca y cortos tiempos de concentración del flujo (Borga et al., 2010, 2014; Destro et al., 2018). De esta forma, la morfometría de la cuenca y la disponibilidad de sedimentos en las laderas y el cauce determinan la susceptibilidad 
de la cuenca a generar eventos torrenciales; entre tanto la intensidad, duración y frecuencia de las condiciones de lluvia determinan las condiciones de amenaza en cuencas susceptibles.

Debido a los cortos tiempos de respuesta y su gran capacidad destructiva, las avenidas torrenciales son una de las amenazas que cobra grandes pérdidas en términos de vidas humanas e infraestructura (S.N.Jonkman, 2005; Marchi et al., 2010; Alfieri et al., 2012; Doocy et al., 2013). En Colombia, de acuerdo con la base de datos del DesInventar se han registrado entre los años 1920 y 2017, 1.643 avenidas torrenciales que han dejado un saldo de 3.277 personas muertas. La región más afectada corresponde a la región Andina con el 72\% de los registros y el $80 \%$ de las víctimas mortales. Tan solo en el departamento de Antioquia se han registrado el 28\% de los eventos torrenciales con un saldo de 1.045 víctimas mortales que representan el 32\% del total, donde el valle de Aburrá es la región más afectada con 187 eventos torrenciales que representan el $11 \%$ del total de eventos, y 303 muertos que representan el 9\% del total de víctimas en Colombia.

Una de las medidas más efectivas para la reducción de las pérdidas asociadas a fenómenos torrenciales detonados por lluvias son los sistemas de alerta temprana (SAT) basados en umbrales empíricos de lluvia (Thiebes and Glade, 2016; Segoni, Piciullo and Gariano, 2018). Cuatro elementos se requieren para un SAT operacional: (i) una evaluación adecuada del riesgo, (ii) la implementación de monitoreo y modelos de predicción, (iii) una estrategia de diseminación de la información simple, sintética y confiable, y (iv) el desarrollo de estrategias de respuesta combinadas con educación y conocimiento en las comunidades (Canli, Loigge and Glade, 2018). (Stähli et al., 2015) clasifican los SAT en (i) sistemas de alerta que están directamente acoplados con sensores e inmediatamente generan una señal cuando se excede un umbral predefinido, la precisión del sistema es alta pero los tiempos de respuesta son muy cortos, (ii) sistemas de alerta que detectan un cambio significativo en variables función del tiempo antes de que el evento ocurra o el umbral se supere, presentan tiempos de respuesta mayores y son utilizados para procesos que presentan un estado progresivo de falla, (iii) sistemas de pronóstico que no utilizan umbrales predefinidos, pero el nivel de amenaza es evaluado por expertos en intervalos de tiempo regulares con base en redes de monitoreo.

Para fenómenos detonados por lluvia como las avenidas torrenciales los umbrales que son incorporados en los SAT son definidos como la cantidad de lluvia requerida para detonar un evento en una determinada área, el umbral mínimo corresponde a la menor cantidad de lluvia por debajo de la cual no se reportan eventos, y el umbral máximo corresponde a la mayor cantidad de lluvia por debajo de la cual siempre se presentan eventos (Reichenbach et al., 1998; Glade, Crozier and Smith, 2000; Gariano, Petrucci and Guzzetti, 2015). Los parámetros más comunes considerados en la definición de umbrales de lluvia son: la cantidad de lluvia acumulada del evento detonante, la lluvia acumulada antecedente en días previos a la ocurrencia del evento y la intensidad-duración del evento de lluvia detonante (Guzzetti et al., 2008). Para la determinación de la lluvia asociada al evento comúnmente se utilizan estaciones de lluvia cerca del sitio de ocurrencia o representativas de la región de análisis (Aleotti, 2004; Capparelli and Tiranti, 2010; Segoni, Piciullo and Gariano, 2018), lo cual representa una gran limitante considerando la variabilidad espacial de la lluvia en terrenos montañosos, como los Andes Colombianos (Poveda et al., 2005). Otras aproximaciones disponibles en los últimos años son los estimados a partir de sensores remotos activos como los radares meteorológicos (Chang and Chiang, 2009; Crosta and Frattini, 2010) y pasivos como los sensores satelitales (Hong and Nasa, 2007; Kirschbaum, Stanley and Simmons, 2015; Cullen, Al-Suhili and Khanbilvardi, 2016), los cuales representan la distribución espacial a resoluciones gruesas sin embargo con mayor representatividad que las estaciones de lluvia, y a escalas temporales con gran incertidumbre 
para resoluciones diarias o de mayor detalle, las cuales son las que generalmente se requieren en este tipo de análisis. Finalmente es importante también considerar que los valores obtenidos de dichos sensores remotos no son mediciones directas, sino que corresponden a estimaciones a partir de la reflectividad medida o de la temperatura de brillo.

Los primeros estudios en Antioquia sobre avenidas torrenciales surgen debido al evento torrencial del 14 de abril de 1988 en la quebrada La Ayurá del municipio de Envigado (Antioquia, Valle de Aburrá) (Caballero and Mejía, 1988; Florez and Parra, 1988). Posteriormente se realizan estudios en las décadas de los 90 por (Hermelin, Mejia and Velasquez, 1992; Cadavid and Hermelín, 2005; González, Chavez and Hermelin, 2005; Piedrahita and Hermelín, 2005). Y recientemente (Caballero, 2011) describe y analiza la susceptibilidad por avenidas torrenciales en el Valle de Aburrá.

$\mathrm{Y}$ aunque diferentes estudios han propuesto umbrales de lluvia en Colombia (Terlien, 1998); Mayorga 2003; Moreno et al., 2006; Salazar, 2011) y en el Valle de Aburrá (Aristizabal et al., 2011; Aristizábal et al., 2010, Echeverri \& Valencia, 2004) como detonantes de movimientos en masa, hasta el momento no se han propuesto umbrales para fenómenos tipo avenidas torrenciales. El presente artículo utiliza el inventario de registro de avenidas torrenciales y series de lluvia con información horaria para proponer umbrales de lluvia como detonante de avenidas torrenciales en el Valle de Aburrá. Para lo cual se utiliza el método propuesto por (Jan et al., 2017) denominado Índice de Lluvia Detonante (RTI por sus siglas en inglés), el cual considera tanto la lluvia detonante en términos de intensidad, como la lluvia antecedente acumulada en los días previos al evento de lluvia. El método permite proponer umbrales de lluvia críticos que pueden ser implementados como parte de un sistema de alerta temprana para reducir las pérdidas en comunidades localizadas en áreas susceptibles a este tipo de fenómenos.

\section{Zona de estudio}

El Valle de Aburrá, localizado en la Cordillera Central del norte de los Andes Colombianos, tiene un área de $1.326 \mathrm{~km}^{2}$, y una longitud aproximada de $65 \mathrm{~km}$. Morfológicamente es descrito (Arias, 1995) como una depresión con orientación sur-norte de fondo plano limitada por respaldos laterales muy inclinados en roca y cubiertos en la parte media y baja por depósitos de vertiente tipo flujo de escombros y lodos (Figura 1). El fondo del valle varía entre 1000 y 3000 msnm hacia su nacimiento. Tres grandes sectores pueden identificarse en el valle: la parte central que consiste en un valle amplio, mientras que los sectores norte y sur consisten en un estrecho valle asimétrico limitado por vertientes con fuertes pendientes.

Las condiciones climáticas son típicas de ambientes tropicales húmedas, con una temperatura promedio de $22^{\circ} \mathrm{C}$ y humedad relativa del $70 \%$. Las condiciones de lluvia son bimodales, con máximas de lluvia en los meses de mayo y octubre. Los promedios de lluvias anuales varían desde $1.400 \mathrm{~mm}$ en la parte central hasta los $2.700 \mathrm{~mm}$ en el norte y sur del valle.

Adicionalmente el Valle de Aburrá corresponde a una subregión de Antioquia que concentra el 59\% (3.3 millones de habitantes) de la población departamental en tan solo 1,8\% del territorio departamental, y lo conforman 10 municipios, donde el 95\% de la población es urbana y se localiza en el $26 \%$ del territorio. Los municipios más densamente poblados son Medellín (2.2 millones), Bello (372.000) e Itagüí (230.000) (DANE, 2005).

Lo anteriormente descrito presenta al Valle de Aburrá como un escenario altamente vulnerable a sufrir afectaciones por avenidas torrenciales, debido a sus condiciones geomorfológicas y a la creciente y acelerada ocupación urbana de terrenos susceptibles (Aristizabal \& Yokota S, 2008) 
Figura 1. Localización del Valle de Aburrá y ocurrencia de avenidas torrenciales entre los años 1934 y 2017. Fuente: Propia

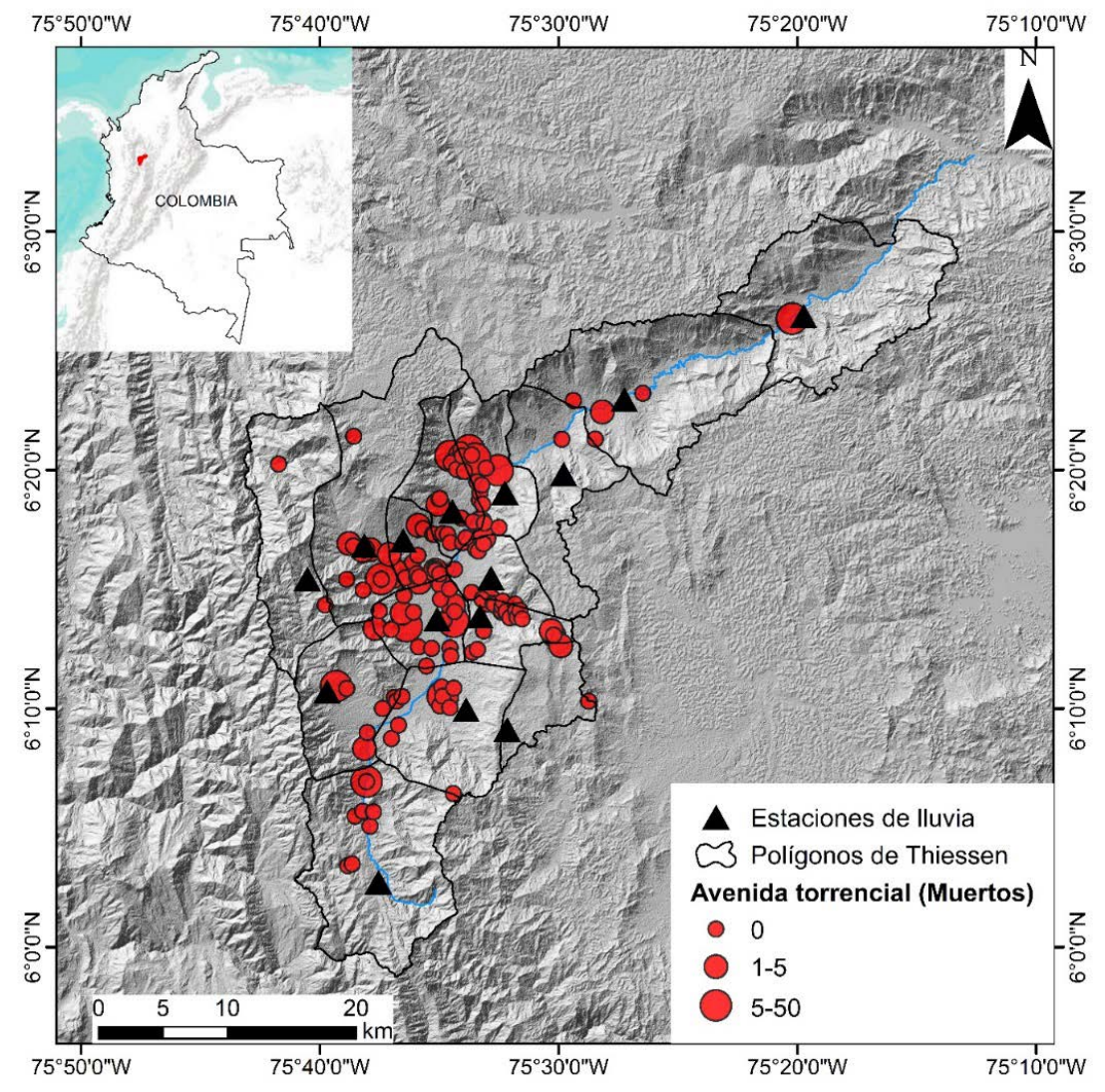

\section{Datos y metodología}

Para la definición de umbrales de lluvia como detonantes de avenidas torrenciales en el Valle de Aburrá se utilizó el método del Índice de Lluvia Detonante (RTI por su siglas en inglés) propuesto por (Jan et al., 2017) del SWCB (Soil \& Water Conservation Bureau) y el COA (Council of Agriculture), institutos encargados de la prevención y mitigación de riesgo por fenómenos torrenciales en Taiwán. La Figura 2 señala esquemáticamente el procedimiento para estimar el Índice de Lluvia Detonante. El RTI se define como el producto entre la intensidad horaria del evento de lluvia que detona la avenida torrencial y la lluvia acumulada antecedente durante un tiempo t:

$$
R T I(t)=I(t) * R t(t)
$$

I(t) es la intensidad del evento de lluvia detonante en un tiempo $t$, medida en milímetros por hora $[\mathrm{mm} / \mathrm{h}]$ y Rt( $\mathrm{t})$ se define matemáticamente por la expresión:

$$
R t(t)=R(t)+\sum_{i=1}^{n} \alpha^{i} R i
$$

El término $\mathrm{R}(\mathrm{t})$ es la lluvia acumulada precipitada durante el evento de lluvia detonante y la sumatoria de la lluvia acumulada antecedente $\mathrm{R}$ de un número $n$ días previos a la ocurrencia de la avenida torrencial. El $\alpha$ describe un factor de peso para los días anteriores al evento, para el cual los autores recomiendan tomar 0.8 (Jan et al., 2017). Para el presente estudio se consideraron diferentes días de 
lluvia acumulada antecedente tomando $n$ valores desde 1, 3, 7 y 15 días.

Debido a que el método requiere identificar los eventos de lluvia detonantes y no detonantes de avenidas torrenciales, se debe definir arbitrariamente un umbral como evento de lluvia. Para el presente estudio se estudiaron diferentes umbrales. Umbrales con valores pequeños de lluvia generan un gran número de eventos de lluvia detonantes sin la ocurrencia de avenidas torrenciales. Por el contrario, umbrales con valores altos generan muy pocos eventos de lluvia detonante, desestimando probablemente eventos que efectivamente generaron avenidas torrenciales. Con esta consideración se optó por un valor intermedio de $20 \mathrm{~mm}$ en una hora, es decir todo evento de lluvia que supere esta intensidad se considera un evento de lluvia detonante para el presente estudio.

Para la información de precipitación se utilizaron 15 estaciones de lluvia localizadas dentro del Valle de Aburrá (ver Figura 1 y Tabla 1), propiedad de Empresas Públicas de Medellín (EPM), y con resolución temporal horaria entre los años 1994 y 2016. La distribución espacial de la lluvia se realizó a partir del método de interpolación con polígonos de Thiessen. De esta forma cada estación tiene un área de influencia, determinada por los polígonos de Thiessen, donde se asume que la precipitación es homogénea en toda el área del polígono.

Para las avenidas torrenciales se compiló el registro del inventario de desastres denominado DesInventar (www.desinventar.org) para el Valle de Aburrá entre los años 1994 y 2017, con un total de 155 avenidas torrenciales registradas (ver Figura 1). El DesInventar no presenta una localización espacial en coordenadas, sino que describe en tres niveles espaciales el sitio de ocurrencia. Con dicha información espacial del total de 155 eventos registrados, solo 97 registros de avenidas torrenciales se lograron ubicar con precisión. Posteriormente, cruzando la fecha de ocurrencia del evento y las series de lluvia a partir de 1994 se obtiene un total de 48 eventos torrenciales ubicados con precisión entre los años 1994 y 2016. Estos datos poseen una limitación ya que el inventario de avenidas torrenciales no registra la hora de la ocurrencia del evento, solo registra el día, por lo tanto, la hora de ocurrencia fue asignada de acuerdo con el evento de mayor intensidad registrado el día de ocurrencia en la estación más cercana.

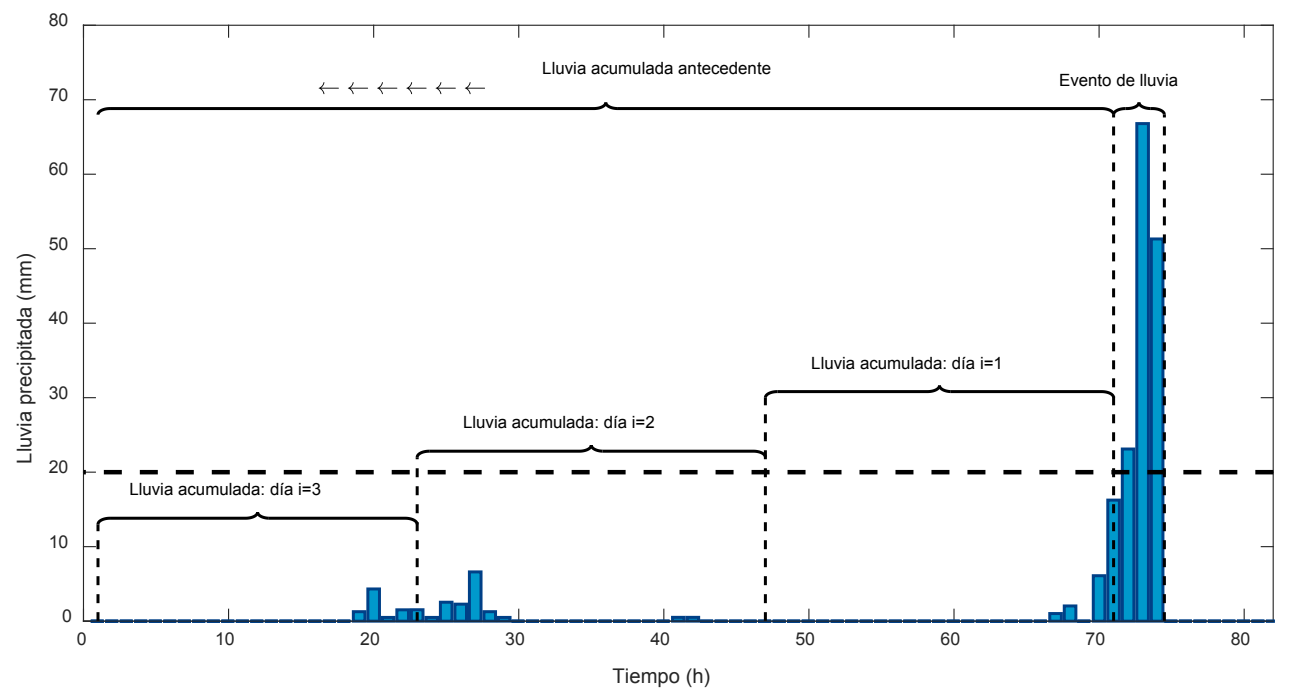


TABLA 1. RESUMEN DE DATOS PARA LAS 15 ESTACIONES DE LLUVIA UTILIZADAS EN EL ANÁLISIS

\begin{tabular}{|c|c|c|c|c|c|c|c|}
\hline $\begin{array}{l}\text { Código } \\
\text { estación }\end{array}$ & $\begin{array}{l}\text { Nombre } \\
\text { estación }\end{array}$ & Latitud $\left({ }^{\circ}\right)$ & $\begin{array}{l}\text { Longitud } \\
\left({ }^{\circ}\right)\end{array}$ & $\begin{array}{l}\mathrm{N}^{\circ} \text { avenidas } \\
\text { torrenciales }\end{array}$ & $\begin{array}{l}\text { Precipitación } \\
\text { media por } \\
\text { hora }(\mathbf{m m})\end{array}$ & $\begin{array}{c}\text { Desviación } \\
\text { estándar } \\
(\mathrm{mm})\end{array}$ & $\begin{array}{l}N^{\circ} \text { eventos de } \\
\text { lluvia }(D>1 \mathrm{~h} \\
\& \mathrm{I}>20 \mathrm{~mm})\end{array}$ \\
\hline 2701114 & Cucaracho & 6,284253 & $-75,60852$ & 17 & 0,196 & 1,623 & 116 \\
\hline 2701115 & Astillero & 6,257315 & $-75,675442$ & 2 & 0,251 & 1,516 & 191 \\
\hline 2701517 & Medellín & 6,2292 & $-75,5849$ & 19 & 0,163 & 0,943 & 61 \\
\hline 2701122 & El Convento & 6,330509 & $-75,496641$ & 1 & 0,187 & 1,439 & 88 \\
\hline 2308023 & Las Palmas & 6,152209 & $-75,536028$ & 2 & 0,255 & 1,378 & 140 \\
\hline 2701036 & Caldas & 6,045471 & $-75,625877$ & 9 & 0,282 & 1,520 & 183 \\
\hline 2701038 & $\begin{array}{c}\text { San Antonio } \\
\text { de Prado }\end{array}$ & 6,178634 & $-75,661738$ & 6 & 0,244 & 1,413 & 181 \\
\hline 2701045 & $\begin{array}{l}\text { Planta Villa } \\
\text { Hermosa }\end{array}$ & 6,257942 & $-75,54725$ & 8 & 0,191 & 1,012 & 70 \\
\hline 2701046 & San Cristóbal & 6,280647 & $-75,635665$ & 14 & 0,197 & 1,162 & 121 \\
\hline 2701950 & Manantiales & 6,317695 & $-75,537506$ & 20 & 0,259 & 1,709 & 221 \\
\hline 2701056 & Girardota & 6,382676 & $-75,454169$ & 4 & 0,181 & 0,947 & 67 \\
\hline 2701057 & Barbosa & 6,441035 & $-75,329066$ & 2 & 0,244 & 1,102 & 82 \\
\hline 2701481 & Pedregal & 6,304601 & $-75,574303$ & 18 & 0,162 & 1,285 & 73 \\
\hline 2701485 & Gerona & 6,231344 & $-75,554944$ & 22 & 0,176 & 1,644 & 86 \\
\hline 2701093 & Ayurá & 6,166051 & $-75,564698$ & 11 & 0,220 & 1,240 & 105 \\
\hline
\end{tabular}

De esta forma, y considerando el umbral de 20 $\mathrm{mm}$ de lluvia previamente definido, se establece el número de eventos de lluvia potenciales de desencadenar un evento torrencial, los cuales corresponden a todos aquellos eventos de lluvia que superen el umbral.

Para cada evento de lluvia se estima el RTI y de acuerdo con el inventario de avenidas torrenciales se identifican los eventos de lluvia que efectivamente generaron avenidas torrenciales.

\section{Resultados}

La Figura 3 presenta la distribución anual de los registros de avenidas torrenciales en el Valle de Aburrá entre los años 1994 y 2016, con el número de víctimas registradas. En términos generales se observa un aumento en el número de eventos y víctimas entre los años 2005 y 2011, y un descenso hasta el año 2015. El 2008 corresponde al año con el mayor número de registros de avenidas torrenciales (17) y el segundo año con el mayor número de víctimas registradas (34), solamente superado por el año 2005, cuando se registraron 40 víctimas en tan solo un evento ocurrido el 6 de octubre en la quebrada El Barro del municipio de Bello.

En el análisis de las series de lluvia para el Valle de Aburrá, teniendo en cuenta la definición de evento de lluvia (D> $1 \mathrm{~h} \& \mathrm{I}>20 \mathrm{~mm}$ ), se obtuvo un total de 1.784 eventos de lluvia entre los años 1994 y 2016. De este total se reportan 6 eventos (0.33\%) con 3 horas de duración, 134 (7.53\%) con dos horas de duración, y 1.642 (92.14\%) con 1 hora de duración. La Figura 4 presenta el número de eventos de lluvia anual identificados en las 15 estaciones de lluvia analizadas comparado con el total de registros 
de avenidas torrenciales en el Valle de Aburrá. Se observa un mayor número de picos entre los años 2002 y 2011, este último el año con el mayor número de eventos (131) que corresponden al 7\% del total de eventos, seguido por el año 2008 con 114 eventos, año en el cual se presenta el mayor número de registros de avenidas torrenciales. Sin embargo, no se observa una relación directa entre el número de eventos de lluvia y la ocurrencia de avenidas torrenciales. Años como el 2011, 2003, 2006 y 2016 que registran eventos de lluvia por encima del valor medio presentan muy pocos registros de avenidas torrenciales.

Para cada uno de los 1.784 eventos de lluvia identificados se calculó el RTI correspondiente, y de manera conexa con el inventario de avenidas torrenciales y el área de influencia de cada estación de lluvia se determinó si el evento de lluvia fue detonante de una avenida torrencial. La Figura 5 presenta los eventos de lluvia identificados en las 15 estaciones del Valle de Aburrá con el valor de RTI correspondiente para un número de días de lluvia antecedente (n) de 1, 3, 7 y 15 días. Los puntos en color azul corresponden a los eventos de lluvia con una duración mayor o igual a $1 \mathrm{~h}$ con intensidad mayor a $20 \mathrm{~mm} / \mathrm{h}$ que no registran avenidas torrenciales. En tanto, los puntos negros corresponden a los eventos de lluvia que registran avenidas torrenciales. Los valores del RTI se encuentran entre $400 \mathrm{~mm}^{2} / \mathrm{h}$ para 1 día de lluvia antecedente hasta $85.805 \mathrm{~mm}^{2} / \mathrm{h}$ para 15 días de lluvia antecedente, con valores medios de $1.809 \mathrm{~mm}^{2} / \mathrm{h}$ para 1 día de lluvia antecedente, 2.133 $\mathrm{mm}^{2} / \mathrm{h}$ para 3 días, $2.413 \mathrm{~mm}^{2} / \mathrm{h}$ para 7 días, 2.575 $\mathrm{mm}^{2} / \mathrm{h}$ para 15 días. Los mayores valores de RTI se presentan entre 10.000 y $90.000 \mathrm{~mm}^{2} / \mathrm{h}$ que se presentaron entre los años 2010 y 2016.

Los eventos de lluvia que presentaron registros de avenidas torrenciales corresponden a valores promedios del RTI, entre 3.701 y $4.774 \mathrm{~mm}^{2} / \mathrm{h}$, con un valor extremo de $46.829 \mathrm{~mm}^{2} / \mathrm{h}$ que corresponde al evento del 30 de noviembre del 2010 ocurrido en la zona urbana de La Sierra (Este del Valle de Aburrá) y que registró 50 viviendas afectadas. El evento con más afectaciones presenta un RTI de $3.657 \mathrm{~mm}^{2} / \mathrm{h}$ ocurrido el 21 de agosto de $2008 \mathrm{con}$ un saldo de 102 viviendas afectadas, 1.000 personas afectadas y una persona muerta. Existe una gran cantidad de eventos de lluvia, con valores de RTI por encima de $48.203 \mathrm{~mm}^{2} / \mathrm{h}$ que no tienen registros de avenidas torrenciales.

Figura 3. Ocurrencia anual de avenidas torrenciales y víctimas fatales asociadas en el Valle de Aburrá entre los años 1994 у 2016

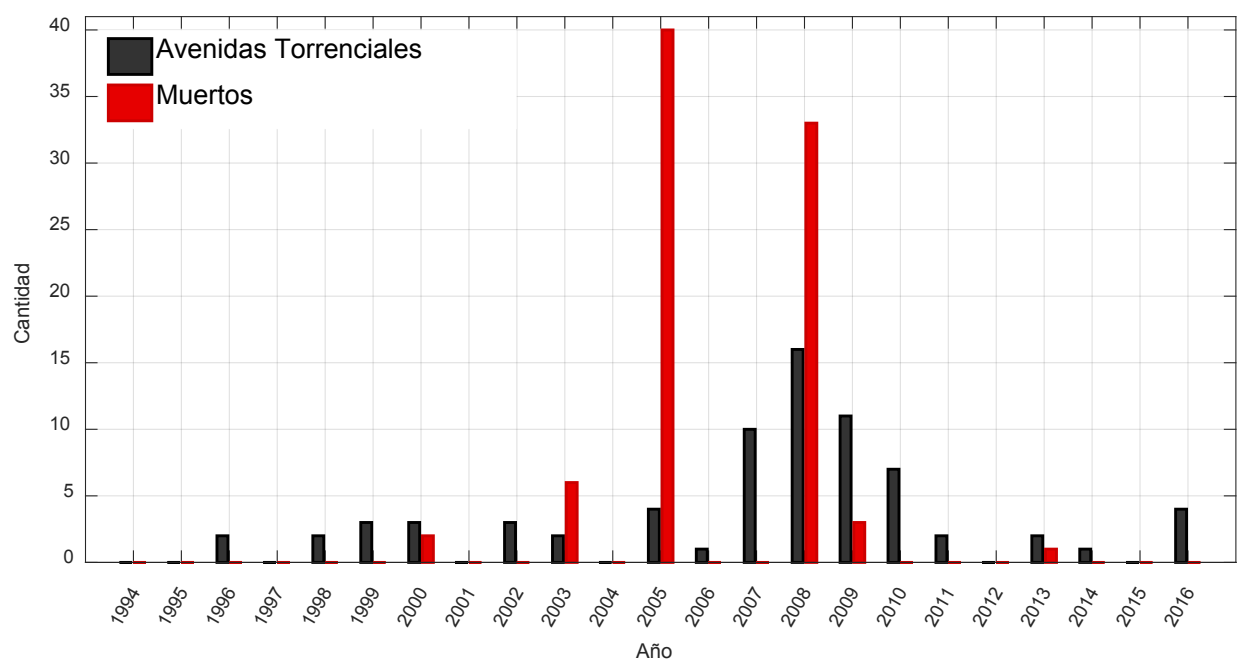


Figura 4. Número de eventos de lluvia ( $\mathrm{D}>1 \mathrm{~h} \& \mathrm{I}>20 \mathrm{~mm}$ ) registrados en las 15 estaciones analizadas comparado con los registros de avenidas torrenciales en el Valle de Aburrá entre los años 1994 y 2016

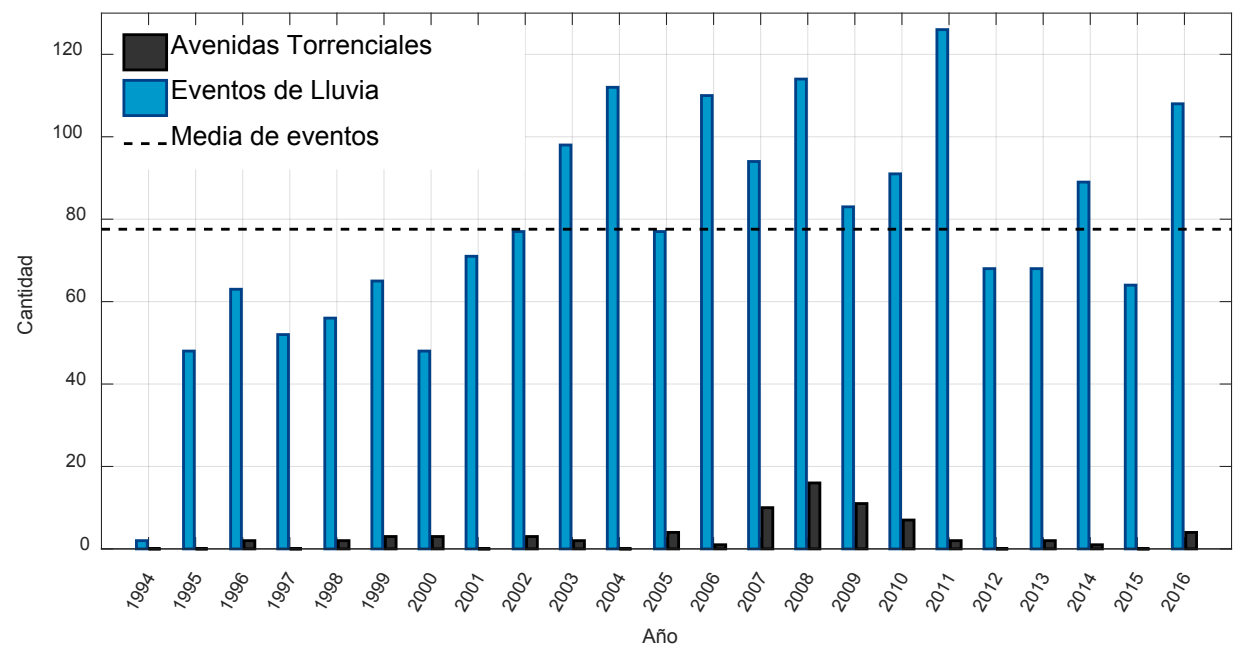

Figura 5. Eventos de lluvia con $D>1 \mathrm{~h} \& \mathrm{I}>20 \mathrm{~mm} / \mathrm{h}$ versus el RTI para diferentes días de lluvia antecedente acumulada, a) $\mathrm{n}=1$ día, b) $\mathrm{n}=3$ días, c) $\mathrm{n}=7$ días, y d) $\mathrm{n}=15$ días. Los puntos en azul corresponden a los eventos sin registro de avenidas torrenciales, y los puntos negros los eventos que tiene asociado la ocurrencia de avenidas torrenciales

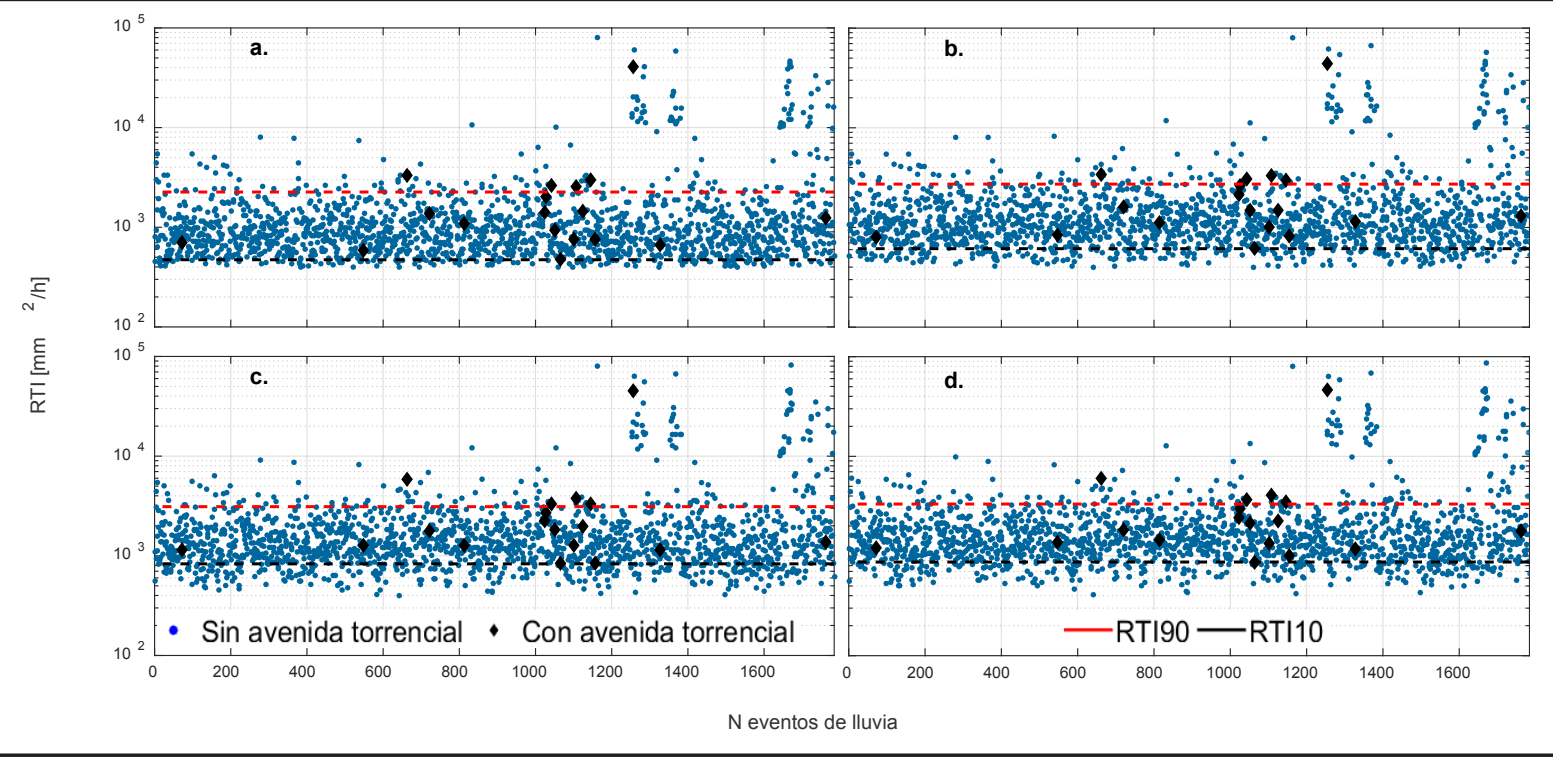

Para la definición de umbrales de alerta se definió el RTI ${ }_{10}$ como el valor del RTI por debajo del cual se encuentra en $10 \%$ de los eventos de lluvia con o sin evento torrencial asociado, y el RTI $\mathrm{R}_{90}$ como el valor del RTI por encima del cual se presenta el $10 \%$ de los eventos de lluvia (percentil 90 de los RTI históricos). Estos umbrales permiten conformar un sistema de alertas amarilla, naranja y roja. La alerta amarilla corresponde aquellos eventos de lluvia que presentan un RTI entre 0 y el RTI $_{10}$, la alerta naranja corresponde a los eventos de lluvia con RTI entre $\mathrm{RTI}_{10} \mathrm{y} \mathrm{RTI}_{90}$, y finalmente la alerta roja para eventos de lluvia con un RTI por encima del $\mathrm{RTI}_{90}$ (Jan et al., 2017). 
Figura 6. Umbrales de lluvia RTI propuestos para avenidas torrenciales en el Valle de Aburra, en función de la intensidad horaria de lluvia y la lluvia antecedente acumulada, considerando eventos de lluvia con $\mathrm{D}>1 \mathrm{~h} \& \mathrm{l}>20 \mathrm{~mm} / \mathrm{h}$ y días de lluvia antecedente acumulada, a) $n=1$ día, b) $n=3$ días, c) $n=7$ días, y d) $n=15$ días

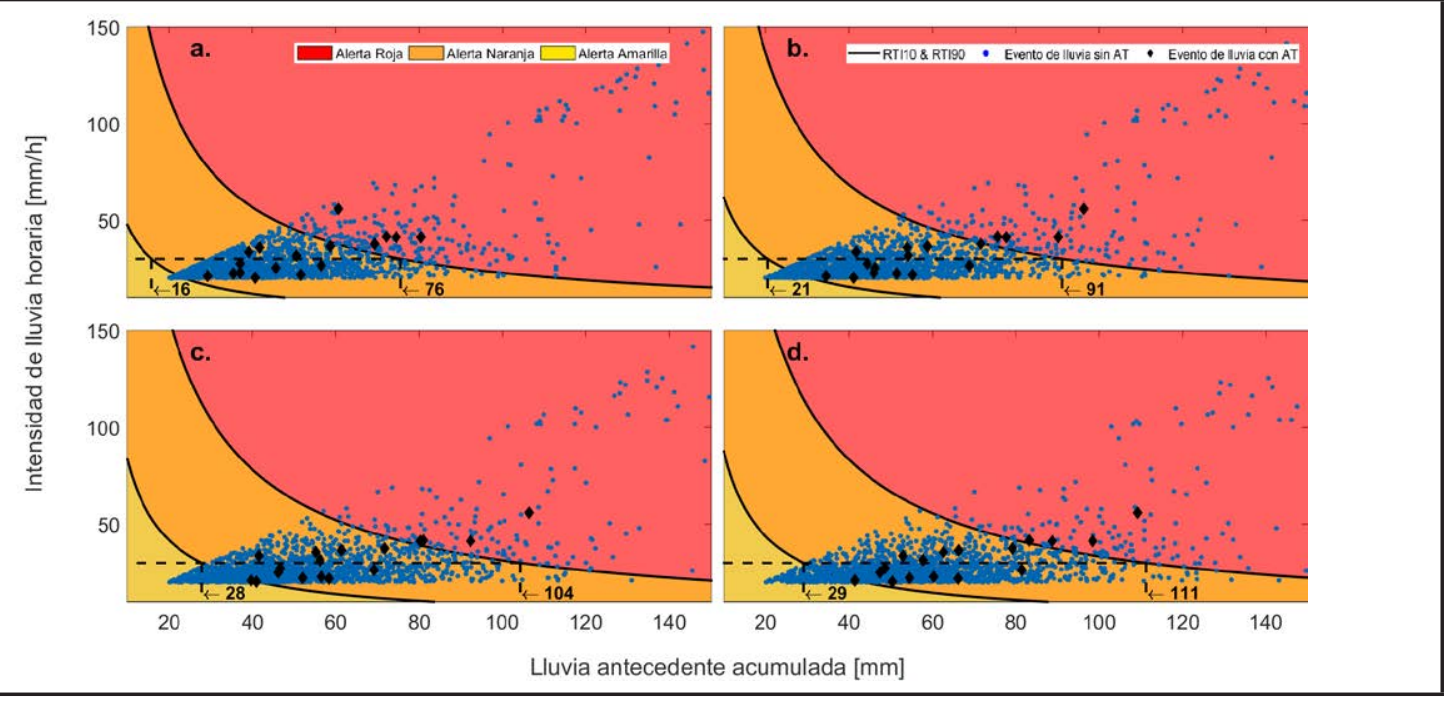

La Figura 5 presenta los eventos de lluvia identificados para el Valle de Aburrá de acuerdo con el valor de $\mathrm{RTI}_{10}$ y el $\mathrm{RTI}_{90}$ para lluvias acumuladas de 1, 3, 7 y 15 días. La metodología encontró que un total de $37.5 \%$ de las avenidas torrenciales útiles fueron asociadas a eventos de lluvia; el porcentaje de eventos con registros de avenidas torrenciales entre el $\mathrm{RTI}_{10}$ y RTI $_{90}$ es de $27 \%$ para lluvia antecedente acumulada de 1, 3, 7 y 15 días; y el porcentaje de eventos de lluvia con registro de avenidas torrenciales es de $10 \%$, por encima del día $\mathrm{RTI}_{90}$ para lluvia antecedente acumulada de 1, 3, 7 y 15 día. Como se puede observar todos los eventos de lluvia con registros de avenidas torrenciales se encuentran para valores por encima del $\mathrm{RTI}_{10}$, y en su mayoría para valores de RTI entre el RTI ${ }_{10}$ y RTI $_{90}$ que corresponde al nivel naranja, y existe un número importante de eventos de lluvia con valores por encima del RTI $_{90}$ que no registran avenidas torrenciales.

Para el sistema de alerta temprana es posible establecer la intensidad de lluvia horaria crítica $I(t)$ de los $n$ días anteriores al evento de lluvia para una lluvia antecedente acumulada $R t(t)$ a partir de la Ecuación: 3:

$$
I(t)=\frac{R T I(t)}{R t(t)}
$$

La Figura 6 presenta los umbrales de lluvia en términos de intensidad horaria de lluvia y lluvia acumulada, resaltando el nivel amarillo para valores inferiores de $\mathrm{RTI}_{10}$, el nivel naranja para valores entre el $\mathrm{RTI}_{10} \mathrm{y} \mathrm{RTI}_{90}$, y el nivel rojo para valores por encima del $\mathrm{RTI}_{90^{\circ}}$. La Tabla 2 presenta los límites de los niveles de alerta en términos del RTI, y el umbral de lluvia crítico considerando la intensidad media de los eventos históricos en la serie entre 1994 y 2008 estimado para este estudio como $I(t)$ de $30 \mathrm{~mm} / \mathrm{h}$ y que son señalados en la Figura 6. Los umbrales observados para el Valle de Aburrá corresponden a valores de lluvia muy bajos. Como se observa en la Tabla 2 y Figura 6, un evento de lluvia con intensidad de $30 \mathrm{~mm} / \mathrm{h}$ presenta valores de lluvia acumulada de 1 día de tan solo $16 \mathrm{~mm}$ para la alerta naranja, 21 $\mathrm{mm}$ de lluvia antencedente acumulada para 3 días, $28 \mathrm{~mm}$ para 7 días, y $29 \mathrm{~mm}$ para 15 días. Para la alerta naranja se presentan valores de lluvia acumulada de $76 \mathrm{~mm}, 91 \mathrm{~mm}, 104 \mathrm{~mm}$ y $111 \mathrm{~mm}$ para 1, 3, 7 y 15 días antecedentes. A medida que la intensidad de la lluvia aumenta, los valores de lluvia acumulada antecedente se reducen ligeramente, hasta valores 
muy altos de intensidad donde la lluvia acumulada antecedente no termina siendo determinante.

\begin{tabular}{|c|c|c|c|}
\hline $\begin{array}{c}\text { Días } \\
\text { antecedentes }\end{array}$ & Límite & $\begin{array}{l}\text { Umbral RTI } \\
{\left[\mathrm{mm}^{2} / \mathrm{h}\right]}\end{array}$ & $\begin{array}{l}\text { Umbral Rt } \\
\text { [mm] }\end{array}$ \\
\hline \multirow{2}{*}{1} & $\mathrm{RTI}_{10}$ & 474 & 16 \\
\hline & $\mathrm{RTI}_{90}$ & 2.268 & 76 \\
\hline \multirow{2}{*}{3} & $\mathrm{RTI}_{10}$ & 616 & 21 \\
\hline & $\mathrm{RTI}_{90}$ & 2.734 & 91 \\
\hline \multirow{2}{*}{7} & $\mathrm{RTI}_{10}$ & 835 & 28 \\
\hline & $\mathrm{RTI}_{90}$ & 3.128 & 104 \\
\hline \multirow{2}{*}{15} & $\mathrm{RTI}_{10}$ & 874 & 29 \\
\hline & $\mathrm{RTI}_{90}$ & 3.337 & 111 \\
\hline
\end{tabular}

Estos bajos valores de lluvia observados para el Valle de Aburrá pueden explicarse por la variabilidad espacial de la misma en en valle, y la subestimacion de la lluvia asociada a eventos torrenciales registrados. Diferentes autores han resaltado la alta variabilidad espacial de la lluvia en ambientes tropicales y de montaña como el valle de Aburrá (Poveda et al., 2005). Adicionalmente la sub estimación de la lluvia puede deberse a que los registros de lluvia que son correlacionados con la ocurrencia de una avenida torrencial se toman de la estación mas cercana al sitio donde se registró el evento torrencial. Lo cual en la mayoría de los casos puede no corresponder a la lluvia detonante, ya que el evento de lluvia representativo de dichos fenómenos torrenciales debe registrarse en la parte superior de la cuenca, y no en la parte inferior, que es donde generalmente se registran las pérdidas y por lo tanto el evento torrencial. Esta situación puede ser corregida asociando entonces el evento de lluvia detonante a los registros de la parte alta de la cuenca. Para el caso del Valle de Aburrá no fue posible realizar dicho ajuste debido a que el gran número de estaciones se localizan en la parte media y baja de las cuencas, teniendo como único registro generalmente la lluvia de la parte baja de la cuenca.

\section{Discusión}

En el presente estudio se analizaron los registros de lluvia a escala diaria y los registros de avenidas torrenciales en el Valle de Aburrá, y se establecieron umbrales de lluvia críticos en función de la intensidad de la lluvia y la lluvia antecedente acumulada, definiendo tres niveles de alerta: amarilla, naranja y roja. Aunque la totalidad de eventos torrenciales registrados se encuentran en los niveles de alerta naranja y roja, un importante número de eventos de lluvia sin registro de avenidas torrenciales se presentan en el nivel de alerta roja.

Adicionalmente se observan valores de lluvia para los umbrales críticos muy bajos. Lo cual puede señalar que los valores de lluvia registrados en las estaciones más cercanas a los sitios donde se registró la avenida torrencial pueden ser no representativos de las condiciones de lluvia que detonaron el fenómeno torrencial. Por lo cual se recomienda adicionar al registro del evento torrencial en la base de datos de desastre la lluvia detonante de la parte alta de la cuenca, de tal forma que obtengan datos más representativos y mejorar en la estimación de los umbrales de lluvia. Sin embargo, es importante resaltar que dicha subestimación de la lluvia corresponde a un escenario conservador, que considerando la capacidad destructiva de las avenidas torrenciales es mucho más recomendable. La definición de umbrales críticos de lluvia con valores máximos o sobreestimando los eventos de lluvia, generaría como resultado umbrales de lluvia con valores mayores conformando escenarios peligrosos y de mayor riesgo.

Finalmente es importante tener claro que aunque el cálculo de umbrales de lluvia es el método más común para evaluar el nivel de alarma en sistemas de alerta temprana por fenómenos hidrometeorológicos, estos umbrales representan solo una simplificación de los procesos físicos que ocurren en el terreno (Reichenbach et al., 1998). La naturaleza de los fenómenos torrenciales implica procesos 
altamente complejos y no lineales que no son considerados en la definición de umbrales.

\section{Referencias}

Aleotti, P. (2004) 'A warning system for rainfall-induced shallow failures', Engineering Geology, 73(3-4), pp. 247-265. doi: 10.1016/j.enggeo.2004.01.007.

Alfieri, L. et al. (2012) 'Operational early warning systems for water-related hazards in Europe', Environmental Science and Policy, 21, pp. 35-49. doi: 10.1016/j.envsci.2012.01.008.

Arias, A. (1995) 'El relieve de la zona central de Antioquia: Un palimsesto de eventos tectónicos y climáticos', Revista Facultad de Ingeniería: Universidad de Antioquia, pp. 9-24.

Aristizábal, E., Gamboa, M., Leoz, F. (2010) 'Sistema de alerta temprana por movimientos en masa inducidos por lluvia para el Valle de Aburrá, Colombia', Revista EIA, 13(c), pp. 155-169.

Aristizábal, E., Gonzalez, T., Montoya, J.D., Velez, J.I., Martinez, H., Guerra, A. (2011) 'Analisis de umbrales empíricos de lluvia para el pronóstico de movimientos en masa en el Valle de Aburrá, Colombia', Revista de la EIA, 15(c), pp. 95-111.

Aristizábal E.; Yokota S. (2008) ‘Evolucion geomorfologica del valle de aburra y sus implicaciones en la ocurrencia de movimientos en masa', Boletin Ciencias de la Tierra, 24, pp. 5-18.

Aulitzky, H. (1980) Derzeitige Sicherheitserwartungen an verschiedene Lawinenschutzmassnahmen. na.

Borga, M. et al. (2010) 'Flash floods: Observations and analysis of hydro-meteorological controls', Journal of Hydrology. Elsevier B.V., 394(1-2), pp. 1-3. doi: 10.1016/j.jhydrol.2010.07.048.

Borga, M. et al. (2014) 'Hydrogeomorphic response to extreme rainfall in headwater systems: Flash floods and debris flows', Journal of Hydrology, 518(PB), pp. 194-205. doi: 10.1016/j.jhydrol.2014.05.022.

Caballero, H. (2011) 'Las avenidas torrenciales :una amenazza potencial en el valle de Aburrá', Revista Gestión y Ambiente, (3), pp. 45-50.

Caballero, H. and Mejía, I. (1988) 'Algunos comentarios acerca del evento torrencial de la quebrada Ayurá (Envigado) del 14-04-88 y sus implicaciones en la evaluación de la amenaza al municipio', 2 Conferencia de riesgos geológicos del Valle de Aburrá.
Cadavid, M. F. and Hermelín, M. (2005) 'El evento del 29 y 30 de Mayo de 2000 en la Estrella y Sabaneta (Antioquia)', in Universidad EAFIT, O. (ed.) Desastres de Origen Natural en Colombia 1979-2004. Medellin.

Canli, E., Loigge, B. and Glade, T. (2018) 'Spatially distributed rainfall information and its potential for regional landslide early warning systems', Natural Hazards. Springer Netherlands, 91(s1), pp. 103127. doi: 10.1007/s11069-017-2953-9.

Capparelli, G. and Tiranti, D. (2010) 'Application of the MoniFLaIR early warning system for rainfall-induced landslides in Piedmont region (Italy)', Landslides, 7(4), pp. 401-410. doi: 10.1007/s10346009-0189-9.

Chang, K. and Chiang, S. (2009) 'Geomorphology An integrated model for predicting rainfall-induced landslides', Geomorphology, 105(3-4), pp. 366-373. doi: 10.1016/j.geomorph.2008.10.012.

Costa, J. E. (1987) 'Hydraulics and basin morphometry of the largest flash floods in the conterminous United States', Journal of Hydrology, 93(3-4), pp. 313-338. doi: 10.1016/0022-1694(87)90102-8.

COUSSOT, P. and MEUNIER, M. (1996) 'Recognition, classification and mechanical description of debris flows', Earth-Science Reviews, 40(3-4), pp. 209-227. doi: 10.1016/0012-8252(95)00065-8.

Crosta, G. B. and Frattini, P. (2010) 'Distributed modelling of shallow landslides triggered by intense rainfall', Natural Hazards and Earth System Science, 3(1/2), pp. 81-93. doi: 10.5194/nhess-3-81-2003.

Cullen, C. A., Al-Suhili, R. and Khanbilvardi, R. (2016) 'Guidance index for shallow landslide hazard analysis', Remote Sensing, 8(10), pp. 1-17. doi: 10.3390/ rs8100866.

DANE (2005) Censo General 2005. Bogotá D.F. Available at: https://www.dane.gov.co/index.php/estadisticaspor-tema/demografia-y-poblacion/censo-general-2005-1.

Destro, E. et al. (2018) 'Coupled prediction of flash flood response and debris flow occurrence: Application on an alpine extreme flood event', Journal of Hydrology. Elsevier B.V., 558, pp. 225-237. doi: 10.1016/j. jhydrol.2018.01.021.

Díez Herrero, A., Laín Huerta, L. and Llorente Isidro, M. (2008) Mapas de peligrosidad por avenidas e inundaciones. Available at: http://www.igme.es/Publi- 
caciones/publiFree/MapasPeligrosidad/Mapas de peligrosidad por avenidas e inundaciones.pdf.

Doocy, S. et al. (2013) 'The Human Impact of Floods: A Historical Review of Events 1980-2009 and Systematic Literature Review', PLoS Currents, (APR 2013). doi: 10.1371/currents.dis.f4deb457904936b07c09 daa98ee8171a.

Echeverri, Oscar; Valencia, Y. (2004) 'Analisis de los deslizamientos en la cuenca de la quebrada La Iguana en la ciudad de Medellín a partir de la integración Lluvia-pendiente-formación geológica', Dyna, 71, pp. 33-45.

Florez, M. T. and Parra, L. N. (1988) 'Avalancha de la quebrada Ayurá del 14 de abril de 1988', II Conferencia sobre riesgos geológicos en el Valle de Aburrá.

FONDO DE PREVENCIÓN Y ATENCIÓN DE EMERGENCIAS (FOPAE) (2011) Cartilla básica de SAT (Sistemas de Alerta Temprana) ante inundaciones. Bogotá.

Gariano, S. L., Petrucci, O. and Guzzetti, F. (2015) 'Changes in the occurrence of rainfall-induced landslides in Calabria, southern Italy, in the 20th century', Natural Hazards and Earth System Sciences, 15(10), pp. 2313-2330. doi: 10.5194/nhess-15-2313-2015.

Glade, T., Crozier, M. and Smith, P. (2000) 'Applying probability determination to refine landslide-triggering rainfall thresholds using an empirical" Antecedent Daily Rainfall Model"', Pure \& Applied Geophysics. Springer, 157(6-8), p. 1059.

González, J. L., Chavez, O. A. and Hermelin, H. (2005) 'Aspectos geomorfológicos de la avenida torrencial del 31 de enero de 1994 en la cuenca del río Fraile y sus fenómenos asociados', Desastres de Origen Natural En Colombia, 1979-2004, p. 135.

Guzzetti, F. et al. (2008) 'The rainfall intensity-duration control of shallow landslides and debris flows: An update', Landslides, 5(1), pp. 3-17. doi: 10.1007/ s10346-007-0112-1.

Hermelin, M., Mejia, O. and Velasquez, R. E. (1992) 'Erosional and depositional features produced by a convulsive event, San Carlos, Colombia, September 21, 1990', Bulletin of the International Association of Engineering Geology-Bulletin de l'Association Internationale de Géologie de l'Ingénieur. Springer, 45(1), pp. 89-95.

Hong, Y. and Nasa, F. A. (2007) 'Satellite Remote Sensing for Landslide Susceptibility Mapping and Landslide Occurrence Prediction on a Global Basis', pp. 5-8.
Hungr, 0. et al. (2001) 'A review of the classification of landslides of the flow type', Environmental and Engineering Geoscience, 7(3), pp. 221-238. doi: 10.2113/gseegeosci.7.3.221.

Hungr, 0. (2001) 'Flow slides and flows in granular soils', Engineering, 9.

Hungr, O., Leroueil, S. and Picarelli, L. (2014) 'The Varnes classification of landslide types, an update', Landslides, pp. 167-194. doi: 10.1007/s10346-0130436-y.

INGEOMINAS - CVC (2001) 'Guia Metodológica para la zonificación de amenazas por fenómenos de remoción en masa-método univariado- y escenarios de riesgo por avenidas torrenicales', Convenio INGEOMINAS -003- CVC 006/2000, p. 154.

Jakob, M. et al. (2006) 'Hydrometeorological thresholds for landslide initiation and forest operation shutdowns on the north coast of British Columbia', Landslides, 3(3), pp. 228-238. doi: 10.1007/s10346006-0044-1.

Jan, C. D. et al. (2017) 'TXT-tool 2.886-1.1: Early warning criteria for debris flows and their application in Taiwan', in Landslide Dynamics: ISDR-ICL Landslide Interactive Teaching Tools: Volume 1: Fundamentals, Mapping and Monitoring, pp. 405-414. doi: 10.1007/978-3-319-57774-6_30.

Kirschbaum, D. B., Stanley, T. and Simmons, J. (2015) 'A dynamic landslide hazard assessment system for Central America and Hispaniola', Natural Hazards and Earth System Sciences, 15(10), pp. 2257-2272. doi: 10.5194/nhess-15-2257-2015.

Marchi, L. et al. (2010) 'Characterisation of selected extreme flash floods in Europe and implications for flood risk management', Journal of Hydrology. Elsevier B.V., 394(1-2), pp. 118-133. doi: 10.1016/j. jhydrol.2010.07.017.

Mayorga, R. (2003) 'Determining rainfall thresholds that trigger landslides in Colombia', Meteorología Colombiana, 7, pp. 157-168.

Ministerio de Medio Ambiente y Desarrollo (2014) ‘Guía Técnica para la formulación de los POMCAS', p. 104.

O'Brien, J. and Julien, P. (1985) 'Physical properties and mechanics of hyperconcentrated sediment flows', in Proc. ASCE HD Delineation of landslides, flash flood and debris flow hazards, pp. 260-279.

Piedrahita, I. and Hermelín, M. (2005) 'La avenida Torrencial del Río Tapartó Antioquia de 1993’, in EAFIT, F. 
E. U. (ed.) Desastres de Origen Natural en Colombia 1979-2004. Medellin, pp. 109-120.

Pierson, T. C. and Costa, J. E. (1987) 'A rheologic classification of subaerial sediment water flows', Reviews in Eingineering Geology, VII, pp. 1-11. doi: 10.1130/ REG7-p1.

PMA-GMA (2007) 'Movimientos en Masa en la Región Andina: Una guía para la evaluación de amenazas', $P u$ blicación Geológica Multinacional, 4(0717-3733), p. 432.

Poveda, G. et al. (2005) 'The Diurnal Cycle of Precipitation in the Tropical Andes of Colombia', Monthly Weather Review, 133(1), pp. 228-240. doi: 10.1175/MWR2853.1.

Reichenbach, P. et al. (1998) 'Regional hydrological thresholds for landslides and floods in the Tiber River Basin (central Italy)', Environmental Geology, 35(2-3), pp. 146-159. doi: 10.1007/s002540050301.

RM Iverson (1997) 'THE PHYSICS OF DEBRIS FLOWS', Reviews of Geophysics, 35(3), pp. 245-296. doi: 10.1029/97RG00426;

S.N.Jonkman (2005) 'Global Perspectives on Loss of Human Life Caused by Floods', Natural Hazards, 34(2), pp. 151-175. doi: https://doi.org/10.1007/ s11069-004-8891-3.

Salazar Gutierrez, L. F. (2011) Determinación de la humedad del suelo para el inicio de movimientos en masa en la región cafetera colombiana con el uso de modelos físicos experimentales. Available at: http://www. bdigital.unal.edu.co/5442/.

Segoni, S., Piciullo, L. and Gariano, S. L. (2018) 'A review of the recent literature on rainfall thresholds for landslide occurrence', Landslides. Landslides, pp. 14831501. doi: 10.1007/s10346-018-0966-4.

Stähli, M. et al. (2015) 'Monitoring and prediction in early warning systems for rapid mass movements', $\mathrm{Na}$ tural Hazards and Earth System Sciences, 15(4), pp. 905-917. doi: 10.5194/nhess-15-905-2015.

Sterling, S. and Slaymaker, O. (2007) 'Lithologic control of debris torrent occurrence', Geomorphology, 86(3-4), pp. 307-319. doi: 10.1016/j.geomorph.2006.09.002.

Takahashi, T. (1991) 'Debris flow', Debris flow, 13, pp. 57-77.

Terlien, M. T. J. (1998) 'The determination of statistical and deterministic hydrological landslide-triggering thresholds', Environmental Geology, 35(2-3), pp. 124-130. doi: 10.1007/s002540050299.

Thiebes, B. and Glade, T. (2016) 'Landslide Early Warning Systems-fundamental concepts and innovative applications', Landslides and Engineered Slopes. Experience, Theory and Practice, (June), pp. 1903-1911. doi: 10.1201/b21520-238.

Vélez, María Victoria; Montoya, Juan David; Moreno, Hernán Alonso; Rhenals, R. L. (2006) 'La Lluvia Y Los Deslizamientos De Tierra En Antioquia: Análisis De Su Ocurrencia En Las Escalas Interanual, Intraanual Y Diaria', Revista EIA, 5(5), pp. 59-69.

\section{PARA CITAR ESTE ARTÍCULO / TO REFERENCE THIS ARTICLE / PARA CITAR ESTE ARTIGO /}

Guerrero Hoyos, L.A.; Aristizábal Giraldo, E. (2019). Estimación y análisis de umbrales críticos de lluvia para la ocurrencia de avenidas torrenciales en el Valle de Aburrá (Antioquia). Revista EIA, 16(32), Julio-Diciembre, pp. 97-111. [Online]. Disponible en: https://doi.org/10.24050/reia.v16i32.1281 